DOI: 10.52837/27382702-2021-34.1-76

\title{
L'EXÉCUTION DE SURÉNA: LES TENDANCES DANS LES RELATIONS DE POUVOIR CHEZ LES PARTHES*
}

\author{
Rouben Manasserian
}

\begin{abstract}
Le capitaine Parthe Suréna, victorieux des légions de Crassus fut exécuté par Orodès aussitôt après sa victoire éclatante. L'examen de ce fait portent à révéler les particularité du développement politique de l'Empire Parthe.
\end{abstract}

Les mots clés: Suréna, Orodès, Arsacides, Parthes, Plutarque, Tiridate, Mithridate III, Rome, relations de pouvoir, G. Kochelenko.

Les auteurs antiques dont les ouvrages sont des sources principales de I'histoire des Parthes traitent de façon passagère les problèmes de l'histoire intérieure politique de l'Empire Arsacide. Ce sont les guerres romanoparthes qui demeurent au centre de leur attention. En plus, en éffleurant les actes politiques des princes Arsacide Trogue Pompée, (Justin), Tacite, Plutarque recourent aux notions idéologiques et morales, telles comme crudelitas, insolencia propres à la mentalité traditionnelle de la police (civitas). Cette particularité de leurs renseignements dissimule les causes des contradictions qui déchiraient périodiquement l'Empire des Arsacides.

D'après le concept de G. A. Kochelenko la lutte politique dans l'Empire parthe fut suscité par les désaccords au sujet de l'attitude envers l'Empire romain, par l'intransigeance entre les partisans de la capitulation devant la Rome et ceux de la lutte opiniâtre ${ }^{1}$. Les premiers furent les couches dominantes des villes hellénistiques de la Mésopotamie méridionale et les nobles Parthes hellénisés alors que les seconds se présentaient comme

\footnotetext{
* The article was submitted on July 29, 2021: The article was reviewed on August 2, 2021.

${ }^{1}$ Kočelenko 1963: 59.
} 
aristocratie clanique des régions orientales ${ }^{2}$. Se fondant sur cette thèse G. Kochelenko interprète la guerre fratricide entre Mithridate III et Orodès II. Déposé par l'aristocratie Parthe Mithridate III (58-57) dut s'enfuir en Syrie romaine. II se rentra aussitôt en Mésopotamie, en trouvant l'appuie dans les cités hellénistiques et parmi eux en Seuleucie du Tigre. G. Kochelenko considère Mithridate comme chef des adeptes de la capitulation devant la Rome, de la reconnaissance de la prépondérance romaine ${ }^{3}$. Cependant il convient d'observer que Kochelenko ne prête pas' attention à l'indication nette de la source, celle de Justin que c'est par la suite de sa cruauté que Mithridate fut détrôné par le "sénat" parthe, une assemblée des chefs claniques. (Propter crudelitatem a Senatu Parthico regno pellitur: - Just., XLII, 4). Justin, donc ne mentionne pas l'attitude défaitiste de Mithridate visà-vis de la Rome, mais indique la cruauté de ce dernier.

Aussi nous paraît-il contestable l'interprétation faite par A. G. Boktchanine qui inverse la suite des faits. "Le calcul de s'appuyer sur la population hellénisée des centres civiques de la Mésopotamie suscita le mécontentement de la noblesse parthe" et "Mithridate fut déposé pour la cruauté"4.

En réalité, la tentative de Mithridate de s'appuyer sur les couches hellénisés de Babylonie et de Seuleucie du Tigre fut déjà entreprise après son détrônement, c'est-à-dire son rapprochement des cercles politiques aux aspirations pro-romaines fut conditionné par la conjoncture politique et de la sorte, ne reflétait pas ses buts initials. Or, ses buts qui lui imposèrent de recourir à la cruauté à l'égard de la noblesse demeurent non élucidés. Prenons en considération que le point de vue qui fait procéder la collision interne chez bles Parthes du désaccord au sujet de l'attitude à l'égard de Rome passe sous silence le fait que Orodès aussitôt après son avènement manifeste lui-même la cruauté. II châtie de mort son excellent capitaine Suréna. Suréna, victorieux des troupes de Mithridate III et des légions de

${ }^{2}$ Kočelenko 1963: 59.

${ }^{3}$ Ibid.

${ }^{4}$ Bokščanin 1966: 43. 
Crassus, chef de l'un de sept clans puissants - membre du "sénat" parthe fut de toute apparence lié au aristocrates semi-nomades orientales de l'Empire. Ce meurtre ne s'enchaîne pas au concept de collision entre les "défaitistes" et les "patriotes".

Ce n'est que Plutarque qui parmi les auteurs antiques a conservé l'information sur le meurtre de Suréna. Selon ses explications le vainqueur de Crassus tomba victime de la jalousie ( $\varphi \theta$ óvoc) royale de sa gloire. ${ }^{5}$ Rappelons-nous que Plutarque considère la jalousie comme motivation générale des actes des protagonistes de ses "Vies". Déjà chez Herodote la jalousie ( $\varphi$ Өóvoc) insite la divinité de poursuivre et de châtier les acteurs pour leurs grandes réussites ${ }^{6}$. II est évident que la version de Plutarque sur la jalousie royale est hors de la réalité politique.

L'exécution de Suréna ne fut pas prise en considération par G. Kochelenko et A.D. H. Bivar. (Ils passent même sous silence ce fait significatif) ${ }^{7}$. Propos du meurtre de Suréna A. G. Boktchanine suppose que "les des données des sources sont tellement maigres qu'il soit impossible d'émettre quelque opinion même la plus générale sur les causes de cette tragédie" ${ }^{1}$. A. N. Sherwin - White attribue une certaine hostilité envers le roi: "l'ambition dynastique"9. Une semblable opinion a été émise par K. Schippmann: "As for Surena, the victor of Carrhae, it, soon cost him his life. Probably fearing, that he would constitute a threat to himself, king Orodès II had him executed"10. "The execution of Suréna, the victor of Carrhae shows the relatively unlimited power of the supreme monarch in Parthia"11.

La dernière assertion est en gros incontestable mais l'affaire de Suréna mérite un examen précis, vue les tendances dans les modifications du

${ }^{5}$ Plutarque 1972. Crass, 33,7. La version de Plutarque de la mort de Suréna par suite de la jalousie d'Orodès fut partagée par Momzenn 1941: 285; Debevoise 1938: 92.

${ }^{6}$ Sobolevsky 1955: 50.

${ }^{7}$ Kočelenko 1963: 58; Bivar 1983: 56.

${ }^{8}$ Bokščanin 1966: 61, note 93.

9 Sherwin-White 1984: 290, "Orodès eliminated his successful marshal on suspicion of dynastic ambition".

10 Schippmann 1987: 528.

${ }^{11}$ Schippmann 1987: 532. 
pouvoir suprême chez les Parthes. II nous convient de présumer que le meurtre de Suréna servit à Orodès de moyens de réaliser des combinaisons politiques de longue portée. L'exécution de Suréna est un acte marquant un coup d'État véritable. II ne serait compris que si l'on prenne en considération les tâches politiques que les Arsacides, chefs militaires et héréditaires du peuple, aux institutions tribales, avaient visé dans leurs rapports avec la haute noblesse clanique. Orodès agit dans l'intention d'instituer l'empire centralisé au pouvoir despotique avec une noblesse servile, un État, en vrai acception de ce mot. Le conflit ne se déroulait pas en forme latente comme on l'estime ${ }^{12}$. Au contraire il revêtu le caractère d'un affrontement ouvert, de la terreur déclenchée par la royauté. Le meurtre de Suréna fut la mesure d'effroi adressée à la haute noblesse, à l'assemblée des chefs des clans. Pour asséner ce coup Orodès choisit en tant que victime Suréna, son serviteur dévoué, mais aussi chef du clan le plus puissant. Ayant, donc s'emparé du trône à l'aide de la noblesse, insurgée contre Mithridate III pour sa cruauté Orodès la prit aussitôt à l'improviste, par le retour à la politique de son prédécesseur. L'exécution de Suréna, non-fondée et arbitraire fut la manifestation de la transformation du pouvoir suprême en pouvoir despotique. La nécessité de la justification de cette mort, c'est à dire de l'argumentation, du recours à l'esprit de ses sujets fut déjà incompatible au caractère nouveau du pouvoir devenu déjà une domination totale et immédiate sur l'individu, sur la personnalité d'un représentant de la classe aristocratique. On réduisait les chefs claniques à l'état des domestiques assujettis. Par son acte Orodès introduisait la peur (metus) en qualité de stimulant de leur soumission. Le sort tragique de Suréna sert de l'illustration de la formule de Trogue Pompée sur les Parthes. " Ils obéissent aux princes non par le respect, mais de la peur, (Principibus meta non pudire parent Just., XLI, III).

La tentative de conjuration de Ornodopate en 51 prouve que les grands ne furent pas résigné complètement à leur nouvelle situation mais gardèrent leur rancune. Dans la formulation, présenté par Trogue Pompée la peur

\footnotetext{
12 Ibid.
} 
figure en qualité de facteur stable du processus de l'exercice du pouvoir. Cette pratique manifestement répressive de l'exercice du pouvoir se reflète dans la doctrine officielle présentée chez Dion Cassius. "Maître, moi le descendant d'Arsace, frère des rois Vologèse et Pacoros, je suis ton esclave, je suis venu vers toi, mon Dieu pour t'adorer comme Mithra. Je serai ce que tu me feras, car tu es mon sort est mon Destin"13. Ces paroles ont été adressées à Néron par le prince Tiridate au moment de son couronnement roi d'Arménie par l'empereur en plein Forum en 66. Tiridate prononça la formule protocolaire, admise à la cour royale arsacide. Cette formule protocolaire qui avait ravie Néron exprime les rapports d'une côté de la domination sans limites et de la soumission sans réserve de celle de l'autre. Le roi est un maître égale à Moira par son rôle à l'égard du sujet. Le maître dispose complètement de son sort, de sa vie et de sa mort. Le sujet, tout d'abord son physique, son corps comme l'indique Plutarque dans sa relation sur la situation des courtisans à la cour de Tigrane, roi d'Arménie, est l'objet du pouvoir. En 66 les relations de pouvoir formulées dans l'allocution de Tiridate ne correspondaient pas déjà aux réalités politiques de l'Empire Parthe. La formule ne devint qu'une formalité. Vers le milieu du ler siècle de $\mathrm{n}$. ère les forces centrifuges prirent le dessus. Les expériences de créer un empire centralisé au pouvoir autocrate et despotique subirent un échec final.

Cet époque particulière fut inaugurée par le coup d'État d'Orodès mais elle était précédée d'une époque de longue crise de l'ordre politique d'une société clano-tribale des nomades Parnes. Cette crise fut engendré par les vastes conquêtes territoriales sous le règne de Mithridate ler. Les chefs Parthes claniques, conducteurs de ses propres forces militaires se transformèrent aussitôt en princes locaux pratiquement indépendants. Les possibilités des Arsacides d'exercer l'influence sur la noblesse se diminuèrent. Donc, la nécessité de régir ces territoires immenses, de les organiser dans une puissance unifiée imposait aux Arsacides la tâche de

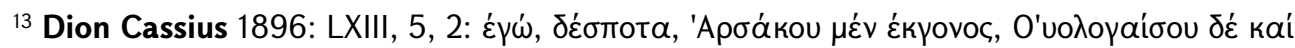

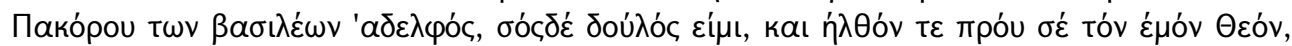

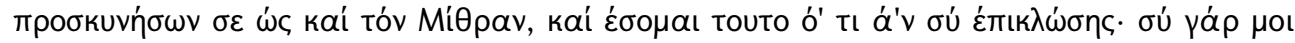

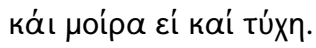


créer un système administratif et centralisé. Pour la réaliser il fallait aux Arsacides de dompter ses compagnons d'arme, les chefs claniques. Cette tâche les poussait inévitablement au conflit, d'autant que ils suivirent les mesures de coersition et de punition qui étaient établis dans une société à l'organisation orientée vers la guerre. La mobilisation des forces face au contre-offensive Seleucide de Démetrios II et Antiochos VII, et des invasions ravageuses des Sakaraukes ajournèrent la résolution du problème. Mithridate II enfin tenta de l'entreprendre. Les sources prouvent l'existence du système satrapal sous son règne. Mais déjà de son vivant ce système ne put empêcher l'explosion de l'Empire et son morcellement durant la période des années 91-70. Sous Phrahate III (70-58) l'Empire surmonta la crise et recouvra son unité. Mithridate III (58-57) reprit la tâche d'instituer autocratie. Orodès semble-t-il la réussit pour un certain delais. La catastrophe Parthe de l'an 38 en guerre contre la Rome de toute évidence réduisit à néan toutes ses entreprises dans le domaine intérieur politique. Les tentatives d'Artabane II (11-38) et des rois rivaux Godarze II et Vardan de rétablir un État despotique et centralisé se brisèrent complètement contre les particularismes de l'aristocratie clanique et foncière.

\section{BIBLIOGRAPHY}

Bivar A. D. H. 1983: The Political History of Iran undee the Arsacides, The Cambridge History of Iran, v. III/I, 21-101.

Bokščanin A. G. 1966: Parfia i Rim", (La Parthie et la Rome), Moscou, 1966, vol. 2, p. 43 (en russe).

Cassii Dionis Cocceianu 1896: Historiarum Romanorum quae supersunt. ed. Boissevain, Berolini.

Debevoise N. C. 1938: A Political History of Parthia, Chicago.

Kochelenko G. A. 1963: Vnutripolititčeskaya borba v Parfii, Vestnik Drevney Istorii, № 3, 56-68 (en russe).

Momzenn Th. 1941: Istoriya Rima, tom. 3, Moskva (Histoire de la Rome) (en russe).

Plutarque 1972: Vies, tome VII, texte établit et traduit par R. Flacelière et É. Chambry.

Schippmann K. 1987: The Arsacide dynasty, Encyclopedia Iranica, Vol. 2, 525-536, New York. 
Sherwin-White A. N. 1984: Roman foreign policy in the East, 168 b. c. to A. D. 1, London.

Sobolevsky I. 1955: Gerodot, Istoriya grecheskoy literaturi. (Herodote, Histoire de la littérature grecque), tom II, Moskva, (en russe).

Rouben Manasserian

Institute of Oriental Studies of the NAS RA, Department of Ancient Orient

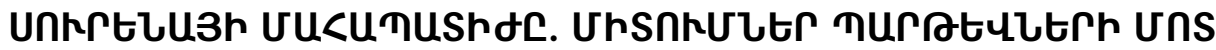

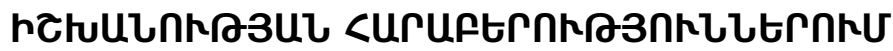

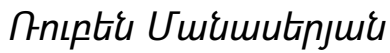

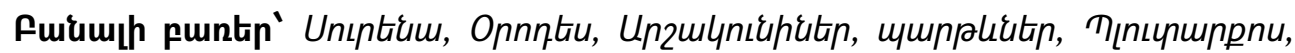

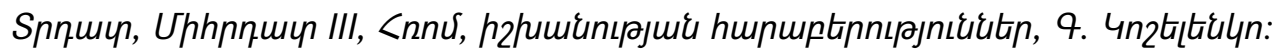

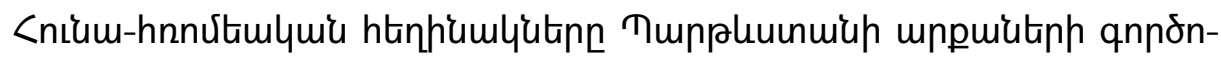

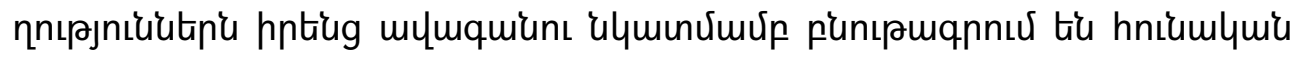

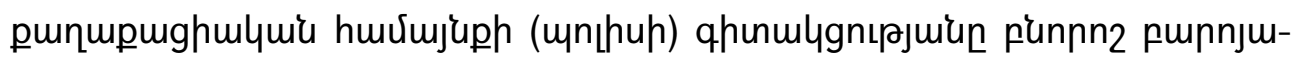

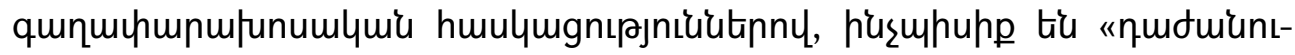

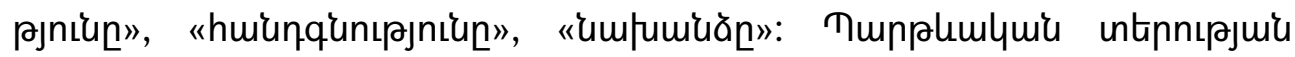

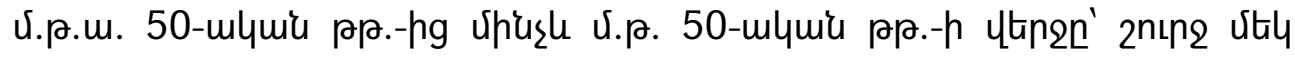

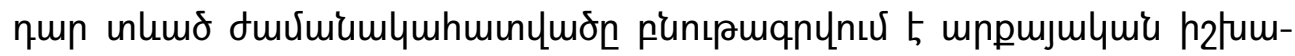

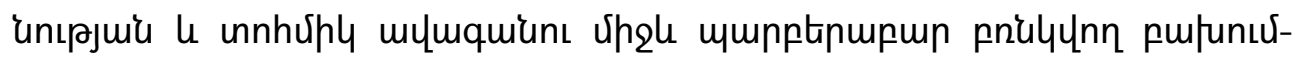

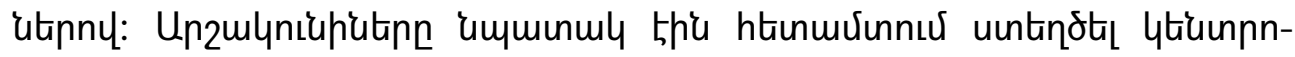

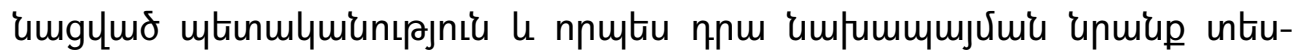

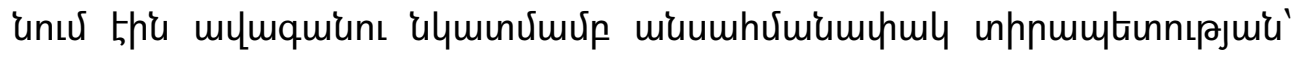

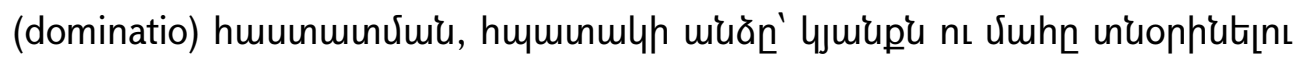

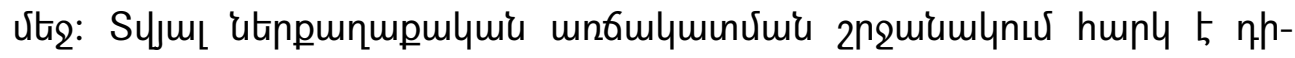

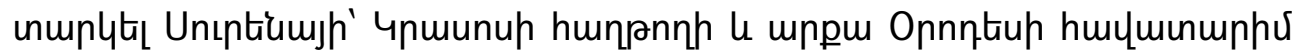

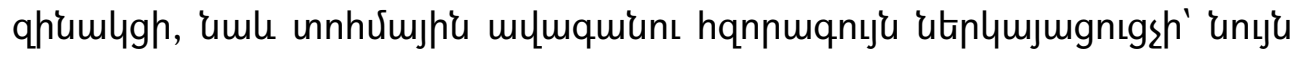

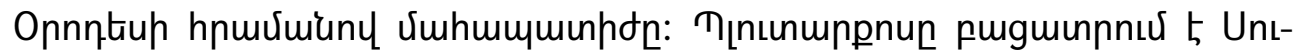

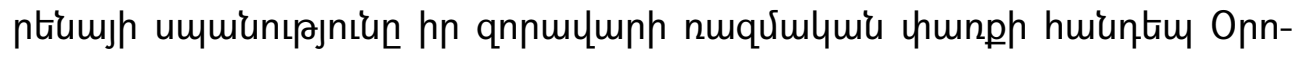

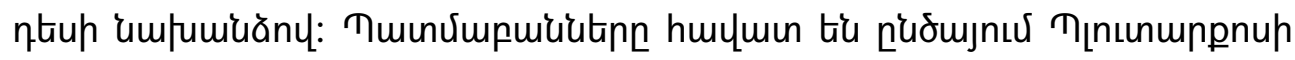




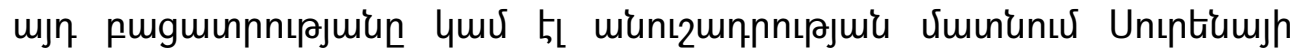

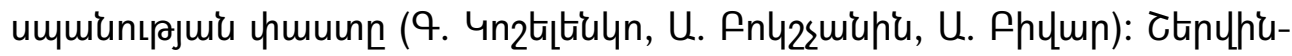

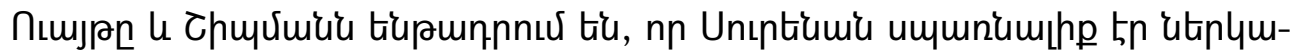

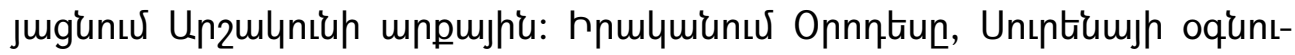

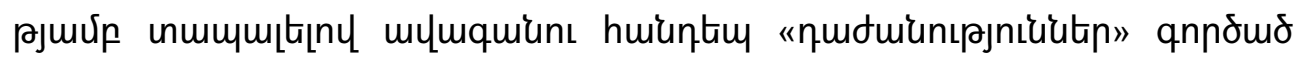

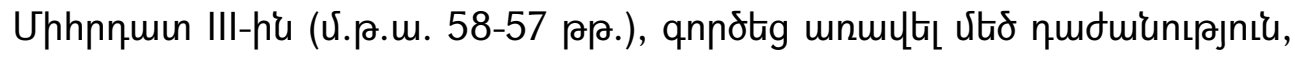

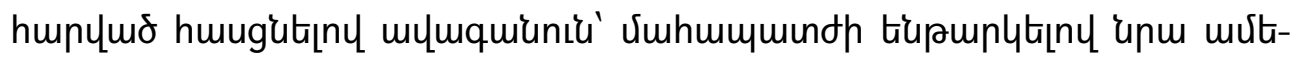

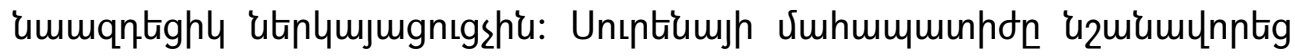

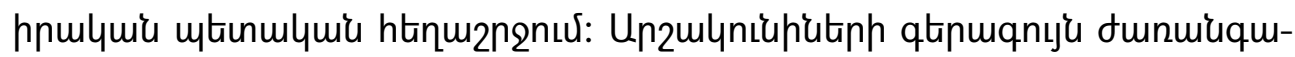

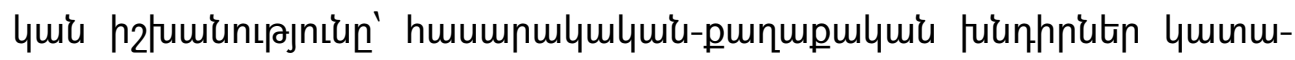

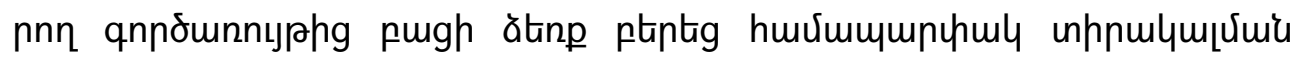

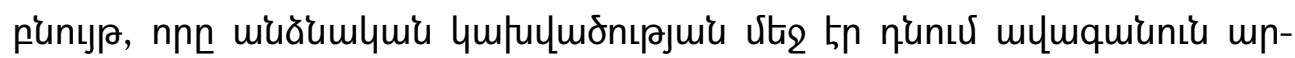

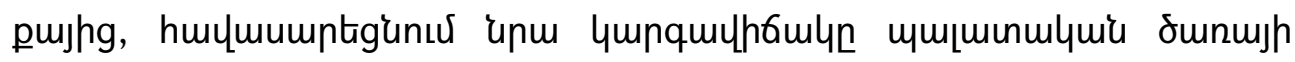

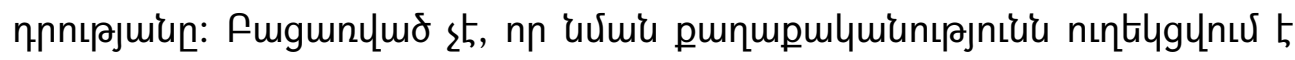

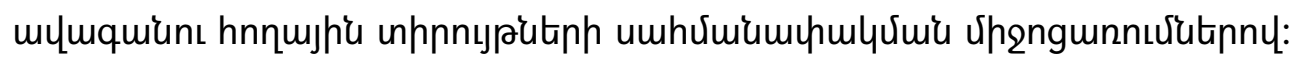

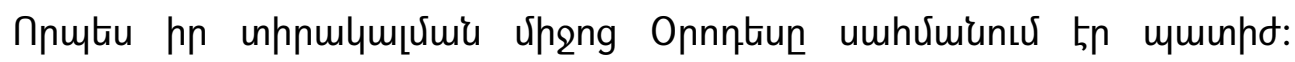

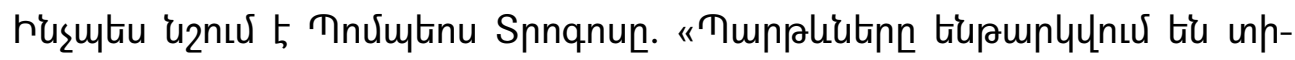

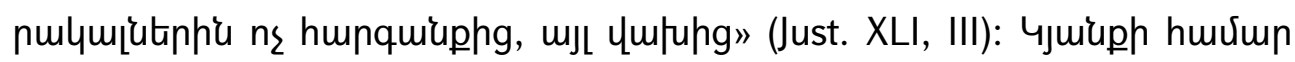

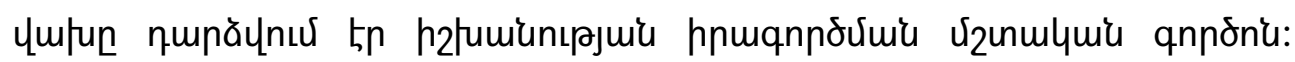

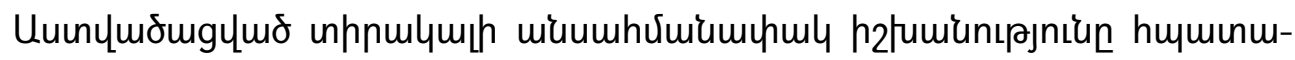

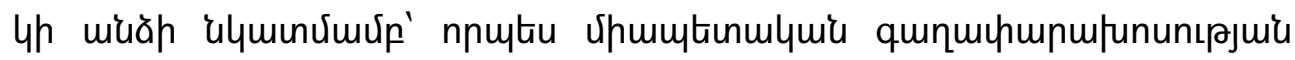

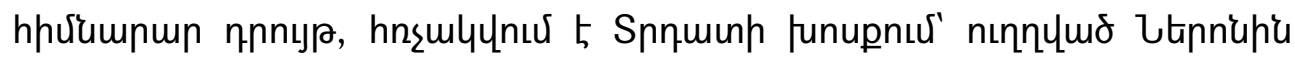
(Dio. Cass, LXIII, 5, 2): Umlumju 66 p. ujn ptiqu unntiu mpunnnulump-

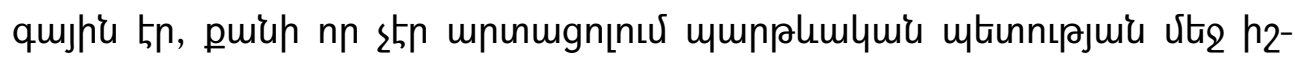

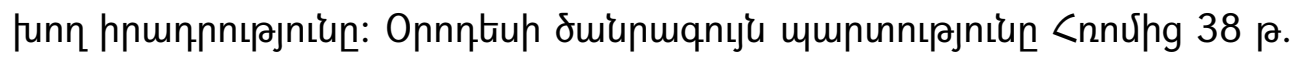

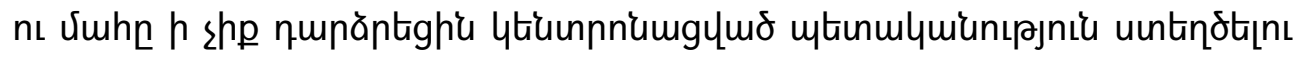
unu quiuptinn: Ujunshtiunl Ununupuiu II-h (11-38 рр.) U hnup ungulyhg

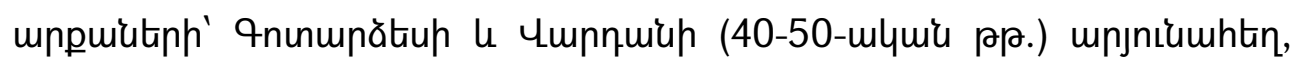

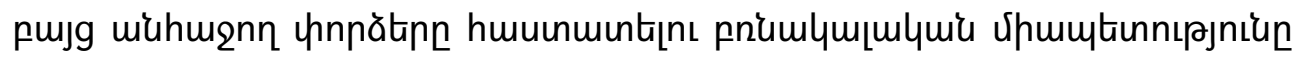

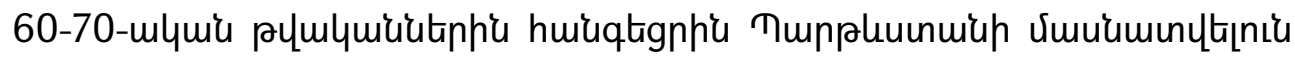

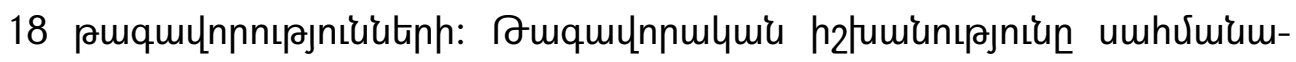

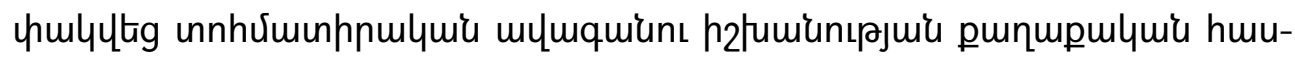
unuunnıpjniüutinny: 